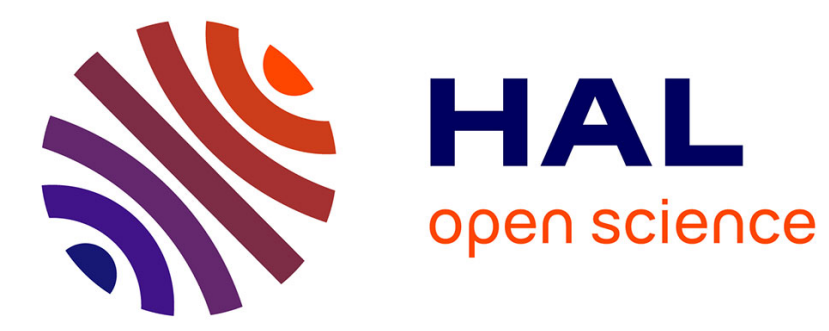

\title{
Visible Light Phase-Shift Rangefinder for Platooning Applications
}

\author{
Bastien Béchadergue, Luc Chassagne, Hongyu Guan
}

\section{To cite this version:}

Bastien Béchadergue, Luc Chassagne, Hongyu Guan. Visible Light Phase-Shift Rangefinder for Platooning Applications. 19th IEEE International Conference on Intelligent Transportation Systems (ITSC), Nov 2016, Rio de Janeiro, Brazil. hal-01378070

\section{HAL Id: hal-01378070 \\ https://hal.science/hal-01378070}

Submitted on 8 Oct 2016

HAL is a multi-disciplinary open access archive for the deposit and dissemination of scientific research documents, whether they are published or not. The documents may come from teaching and research institutions in France or abroad, or from public or private research centers.
L'archive ouverte pluridisciplinaire HAL, est destinée au dépôt et à la diffusion de documents scientifiques de niveau recherche, publiés ou non, émanant des établissements d'enseignement et de recherche français ou étrangers, des laboratoires publics ou privés. 


\title{
Visible Light Phase-Shift Rangefinder for Platooning Applications
}

\author{
Bastien Béchadergue ${ }^{1,2}$, Luc Chassagne ${ }^{2}$ and Hongyu Guan ${ }^{2}$
}

\begin{abstract}
Vehicle positioning and environment awareness are key components in new intelligent transportation systems (ITS) which explains the growing demand in distance sensors. Though several efficient technologies already exist, the growing automation of vehicle requires sensors redundancy. In this paper, we introduce a new, simple and low cost distance measurement scheme using the headlamps and taillights of the vehicles. The following vehicle (FV) emits a periodic signal toward the leading vehicle $(\mathrm{LV})$ which receives, processes and re-emits it. The FV then exploits the phase-shift introduced by this round trip to recover the distance. A complete simulation model is built and tested. This study shows that our system is able to measure distances up to 25 meters with a resolution under 10 centimeters, and up to 30 meters with a 30 centimeters resolution, at a refresh rate of $267 \mathrm{~Hz}$. Even if the regulated optical power and beam limits the range of such a system, it is particularly suited for platooning applications where vehicles are close.
\end{abstract}

\section{INTRODUCTION}

First introduced for adaptive cruise control (ACC) [1], distance sensors are now becoming crucial in most intelligent transportation systems (ITS) since they allow the vehicles to detect and reconstruct the environment in which they operate. Several technologies have been proposed for distance measurement, though they are all based on coherent waves reflected back by the target and then processed in order to recover the distance.

The most famous and developed one is the frequencymodulated continuous-wave (FMCW) radar operating on the $24 \mathrm{GHz}$ or $79 \mathrm{GHz}$ band. It is already commercially used for short-range to long-range ACC but is still too complex and expensive and thus reserved to luxury cars [2]. In addition, severe interference issues might appear as the number of equipped vehicles increases [3]. Direct detection time-offlight (TOF) systems are also widely used. The most common variation is the ultrasound sonar used for short-range distance measurement in park assist systems. These sensors are very cheap but limited in range. Another variation is the LIDAR based on infrared laser. Some systems are able to achieve centimeter resolutions on ranges of several tens of meters over a wide field of view (FOV) using a rotating optical platform [4]. Indirect detection TOF can also be used. It consists in measuring the phase-shift of a periodic signal introduced by the round trip from the system to the target [5]. However, these systems remain complex, expensive and

\footnotetext{
${ }^{1}$ Bastien Béchadergue is with the Vedecom Institute, 77 rue des Chantiers, 78000, Versailles, France (bastien. bechadergue@vedecom.fr)

${ }^{2}$ Luc Chassagne and Hongyu Guan are with the Laboratoire d'Ingénierie des Systèmes de Versailles (LISV), University of Versailles Saint-Quentin, University of Paris-Saclay, 10-12 avenue de l'Europe, 78140, Vélizy, France (luc.chassagne@uvsq.fr, hongyu.guan@uvsq.fr)
}

interferences might again be a growing issue [6]. Other techniques have been proposed such as triangulation. Sub-meters resolutions on ranges of tens of meters have been reported but are achieved at the cost of complex image processing [7]. A variation using the headlamps and taillights has also been proposed [8] but requires modulation frequencies higher than $20 \mathrm{MHz}$ to achieve a 1 meter resolution [9].

Among others, platooning is a typical ITS application for distance sensors. Started in the 60s [10], the research on platooning has gained strong interest over the last decades to increase traffic flow and security. Several projects have been lead to develop this technique [11]. The European SARTRE project achieved in 2012 a fully autonomous, infrastructure independent, 5 to 10 meters platoon lead by a manually driven vehicle. Longitudinal control is achieved using data from both vehicle-to-vehicle (V2V) communication and radar/LIDAR sensors acquisition [12]. V2V communication is achieved through $802.11 \mathrm{p}$ based radio frequency (RF) systems which offer long range and high data rate communication but can suffer from severe degradations caused by interferences in dense traffic scenarios. Visible Light Communication (VLC) has thus been proposed as a complementary technology since it is particularly suited for short-range communication [13].

In the same spirit, this paper presents an inter-vehicle distance measurement system using headlamps and taillights that could be used as a redundant sensor in platooning applications. Indirect TOF by phase-shift measurement is used. However, the non-coherent white light produced by headlamps will be too severely weakened after reflection by the target to be detected by the system. A new scheme is thus proposed to bypass this issue. Simulink simulations show that the resulting system is able to measure distances up to 25 meters with a resolution under 10 centimeters, and up to 30 meters with a 30 centimeters resolution.

The remainder of this paper is organized as follows. The working principles of our distance measurement system are presented in Section II. In Section III, the models used for the optical wireless channel as for our system are presented in details. The numerical results obtained are then developed in section IV. Finally, conclusions and future works are given in Section V.

\section{Measuring Distances Using Vehicle Lighting}

In a platooning configuration, a leading vehicle (LV) is closely followed by a following vehicle (FV) as shown in Fig. 1. In order to adjust its trajectory, the FV must be able to measure its distance to the LV. Here, this function is performed by a modified phase-shift range finder. 


\section{A. Basics of phase-shift range finder}

In order to estimate distances, a phase-shift range finder simply emits a periodic signal at frequency $f$ toward a target. The target reflects back this signal which is received by the system with a phase-shift $\varphi$ proportional to the distance system/target $D$. By measuring the phase-shift $\varphi$, one can simply recover the distance $D$ using:

$$
D=\frac{c}{2 f} \cdot \frac{\varphi}{2 \pi},
$$

where $c$ is the light velocity. The choice of the modulation frequency $f$ is crucial since the non-ambiguity range is $D_{\text {namb }}=c / 2 f$ whereas the distance measurement resolution $\delta D_{\min }$ is defined as:

$$
\delta D_{\min }=\frac{c}{2 f} \cdot \frac{\delta \varphi_{\min }}{2 \pi},
$$

where $\delta \varphi_{\min }$ is the phase measurement resolution. This equation shows that the distance measurement is better in terms of resolution as the phase measurement resolution increases or as the modulation frequency increases. However, increasing $f$ reduces the non-ambiguity range. This tradeoff will be addressed in Section II.C.

\section{B. General operating principle}

Phase-shift distance measurement is usually implemented with radio or infrared monochromatic laser sources that are coherent. The reflected beam is thus strong enough to be detected by the system. However, the white light produced by the FV headlamps is polychromatic and non-coherent so a major part of the optical power is lost after its reflection by the target. To bypass this issue, the LV, instead of only reflecting the signal, can receive, process and re-emit it. This method called Distance Estimation via Asynchronous Phase Shift (DEVAPS) and first presented for radio sensors in [14] allows to restore the energy of the signal so that the echo received by the FV is strong enough to be detected and then processed and compared with the emitted signal to recover the phase-shift and finally the distance. This general operating principle is detailed by Fig. 1 .

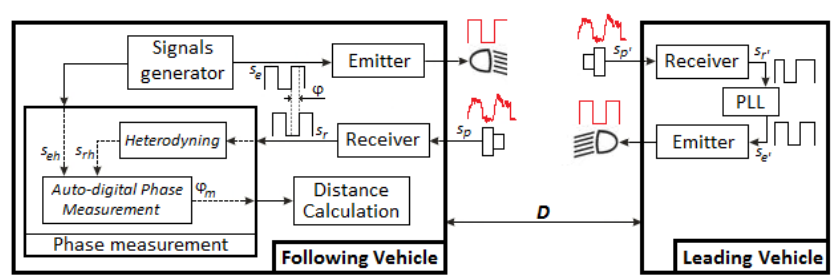

Fig. 1. Block diagram of the visible light phase-shit rangefinder system.

The different steps are as follows: first, the FV emits a square signal $s_{e}$ at frequency $f_{e}$ with its LED headlamps driven by an emitter circuit. A distorted signal $s_{p^{\prime}}$ is then received by a photodetector on the $\mathrm{LV}$ and processed in order to remove the channel noise and recover a square signal $s_{r^{\prime}}$ of frequency $f_{e}$. A PLL may be added in order to recover a $50 \%$ duty cycle square signal $s_{e^{\prime}}$ of frequency $f_{e}$. Note however that the PLL may also generate a signal $s_{e^{\prime}}$ of frequency multiple of $f_{e}$. In this case, the non-ambiguity range issue introduced in Section II.A can be solved. In any case, the square signal $s_{e^{\prime}}$ is emitted by the LV toward the FV with its LED taillights driven by an emitter. The FV receives a distorted signal $s_{p}$ and process it in order to recover a 50\% duty cycle square signal $s_{r}$ of frequency $f_{e}$. At this stage, the FV knows the signal emitted at first $s_{e}$ and the received echo $s_{r}$. These two signals have a phase-shift $\varphi$ proportional to the distance between both vehicles $D$. This phase-shift is then measured using the method detailed in the next section.

\section{Phase-shift measurement technique}

The phase-shift between $s_{e}$ and $s_{r}$ is measured using the auto-digital phase measurement technique [15], [16], illustrated by Fig. 2. The phase difference signal $s_{\varphi}$ is obtained with a simple XOR gate with inputs $s_{e h}$ and $s_{r h}$. $s_{e h}$ and $s_{r h}$ are heterodyned versions of $s_{e}$ and $s_{r}$, that is signals with the same phase-shift but at a lower frequency $f_{i}$ as we will see later. Consequently, $s_{\varphi}$ has a frequency of $2 f_{i} . s_{\varphi}$ is then combined through an AND gate with a gate signal $s_{\text {gate }}$ of period $N / f_{i}$, where $N$ is a non-negative integer corresponding to the number of phase difference pulses taken into account in each measurement for averaging. The gate signal simply allows clock-counting when it is at high level. The resulting signal $s_{\varphi^{\prime}}$ is again combined through an AND gate with a clock signal $s_{\text {clock }}$ of frequency $f_{\text {clock }} \gg f_{i}$, giving the signal $s_{\varphi^{\prime \prime}}$. A counter is then incremented after each rising edge of $s_{\varphi^{\prime \prime}}$ and is reset when the gate signal is at low level. The resulting count $M$ is converted into a phase estimate using the formula:

$$
\varphi_{\text {est }}=2 \pi \cdot \frac{M f_{i}}{N f_{\text {clock }}} .
$$

Equation (1) can then be used to recover the measured distance from $\varphi_{\text {est }}$. Note that when $s_{\text {gate }}$ is at low level, a second parallel channel is enabled to measure the phase difference with the same method so that no phase difference pulse is lost [17].

As mentioned earlier, auto-digital phase measurement is not directly applied to $s_{e}$ and $s_{r}$ but to their heterodyned versions $s_{e h}$ and $s_{r h}$. This technique is used to increase the phase-shift measurement resolution $\delta \varphi_{\min }$ and thus the distance measurement resolution (2) by solving the double tradeoff due to the modulation frequency choice. The first aspect of this tradeoff has been presented in Section II.A. The second aspect is inherent to the auto-digital phase measurement method: $\delta \varphi_{\min }$ depends directly on the counter frequency $f_{\text {clock }}$ such that as the modulation frequency $f_{e}$ increases, $f_{\text {clock }}$ also has to increase in order to keep $\delta \varphi_{\text {min }}$ constant. Heterodyning allows to keep a high modulation frequency while applying the auto-digital phase measurement method to signals of lower frequency, thus limiting the counter frequency. In our case, heterodyning is simply achieved using a D flip-flop with a clock signal $s_{h}$ of frequency $f_{h}$ very close to $f_{e}$. As in Sequential Equivalent Time Sampling, $s_{h}$ can be seen as a sequential sampling 


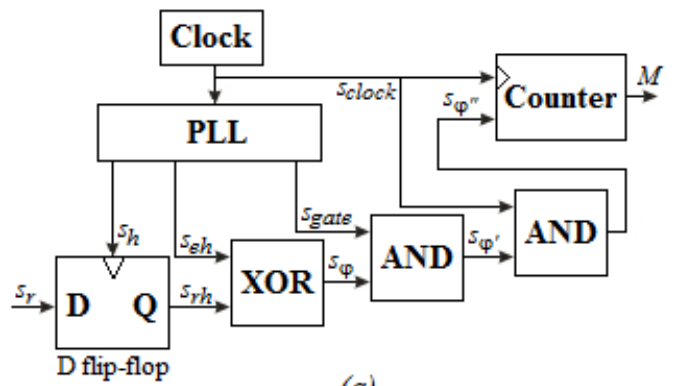

(a)

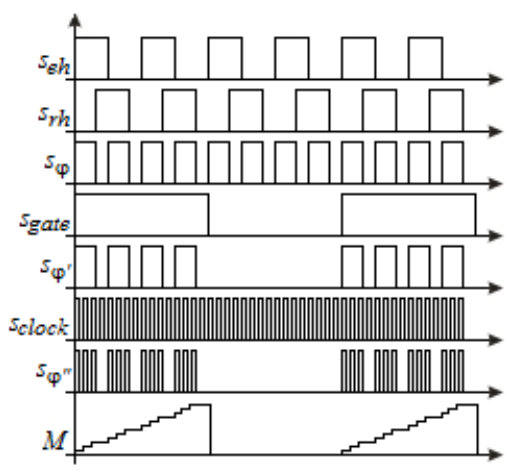

(b)

Fig. 2. (a) Block diagram and (b) timing sequence of the auto-digital phase measurement system.

signal taking $r$ samples per period $1 / f_{e}$. Thus, $f_{h}$ and $f_{e}$ are linked by the relation:

$$
f_{h}=\frac{r}{r+1} \cdot f_{e}
$$

which gives the following intermediate frequency $f_{i}$ :

$$
f_{i}=\frac{f_{e}}{r+1} .
$$

The combination of (3) and (5) gives the measured phaseshift:

$$
\varphi_{\text {est }}=2 \pi \cdot \frac{M f_{e}}{(r+1) N f_{\text {clock }}} .
$$

Finally, the refresh rate $T_{r}$ of the distance measurement is:

$$
T_{r}=\frac{2 f_{e}}{(r+1) N} \text {. }
$$

\section{Error analysis}

The phase measurement error $\delta \varphi_{\text {est }}$ can be derived by differentiating (6), which gives:

$$
\delta \varphi_{\text {est }}=\varphi_{\text {est }}\left[\frac{\delta f_{i}}{f_{i}}+\frac{\delta M}{M}-\frac{\delta f_{\text {clock }}}{f_{\text {clock }}}-\frac{\delta N}{N}\right] .
$$

Each term has a different explanation: $\delta f_{\text {clock }}$ represents the frequency drift of the following vehicle local oscillators caused by temperature variation and age.

$\delta f_{i}$ represents two major error sources. First of all, the signal emitted by the LV $s_{e^{\prime}}$ has in practice a frequency of $f_{e^{\prime}}$ rather close but still different to $f_{e}$ and a duty cycle that may be different to $50 \%$. These distortions are mainly introduced by the processing of the received signal distorted signal $s_{p^{\prime}}$ to recover the square signal $s_{e^{\prime}}$ but may also be caused by the frequency drift of the LV local oscillator. The second source of error is the heterodyning method used. Whatever the sampling rate $1 / r f_{e}$, the edges of the square signal $s_{r}$ may happen between two sample points. For instance, if a rising edge of $s_{r}$ happens between sampling points $n$ and $n+1$, the corresponding rising edge of the transposed signal $s_{r h}$ will happen at sampling point $n+1$ because $s_{r h}$ is synchronized with the sampling signal $s_{h}$. Consequently, a time shift of at most $1 / r$ is introduced by the heterodyning step. This shift can be reduced by increasing $r$ but at the cost of the refresh rate. Fig. 3 illustrates the impact of the choice of $r$ on (a) the distance measurement resolution $\delta D_{\min }$ and (b) the measurement refresh rate $T_{r}$ for different averaging values $N$. All things being equal, $r$ must be at least equal to 1500 in order to have a distance measurement resolution under 10 centimeters. In this case, the refresh rate is $1332 \mathrm{~Hz}$ without averaging.

$\delta M$ represents the counting error induced by the finite counting frequency $f_{\text {clock }}$. A phase-shift pulse duration does not correspond exactly to an integer multiple of the counting period. Consequently, an error of $\pm 1 \max$ is contained in each phase-shift pulse measurement. This error source is mitigated by averaging over $N$ phase-shift pulse measurements. In this case, the max error remains \pm 1 but the intermediate errors are lower. Fig. 3(c) illustrates the impact of the counter frequency choice $f_{\text {clock }}$ on the distance measurement resolution $\delta D_{\min }$ for different averaging values $N$. A counting frequency higher than $50 \mathrm{MHz}$ introduces an error below 2 millimeters which makes this parameter far less critical than $r$, especially if averaging is used. Note however that $T_{r}$ dramatically drops as $N$ increases so this parameter must be chosen carefully.

Finally, $\delta N$ represents the potential non-synchronization between the gate signal $s_{\text {gate }}$ and the phase-shift signal $s_{\varphi}$. If $s_{\text {gate }}$ changes to high level slightly after a rising edge of $s_{\varphi}$, a part of the corresponding phase-shift pulse will be lost. Such non-synchronization can appear with the drift of the oscillator generating $s_{\text {gate }}$. However, if $s_{\text {gate }}$ is generated from the clock signal $s_{\text {clock}}$, this source of error can be mitigated.

\section{Simulation Model}

The Simulink model simulates a platoon of two vehicles as in Fig. 4. The LV and FV have respectively a receiver, composed of an array of photodiodes (PD), on the rear and front and uses both taillights and both headlamps as transmitters. Transmitters and receivers of both vehicles are in the same plane. In addition, a continuous line-of-sight (LOS) is considered between each transmitter/receiver pairs but crosstalk is ignored.

The LOS channel model used is the classical path-loss Additive White Gaussian Noise (AWGN) channel model [18], where the output current $Y(t)$ produced by the PD is linked with the transmitted optical power $X(t)$ by:

$$
Y(t)=\gamma X(t) \otimes h(t)+n(t),
$$




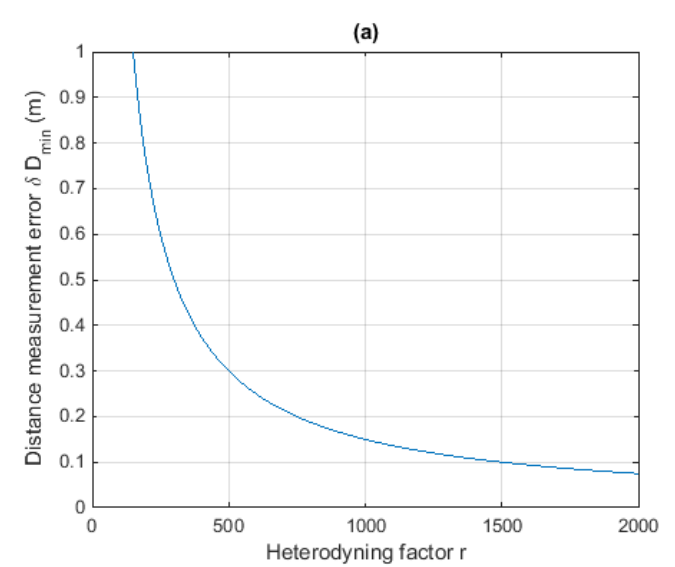

(b)

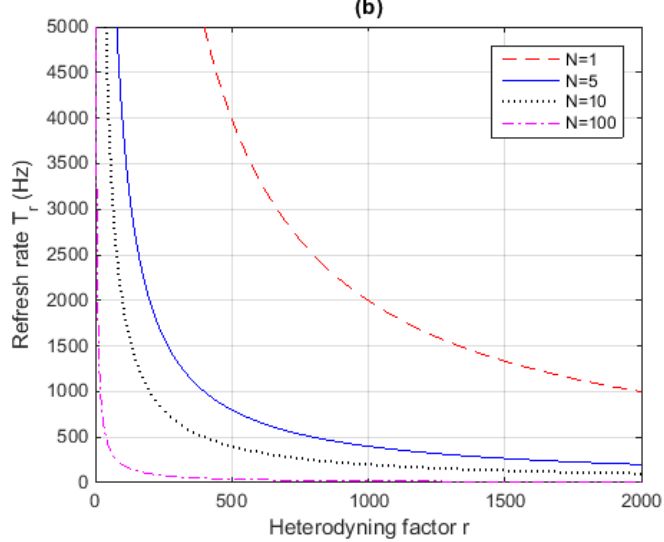

(c)

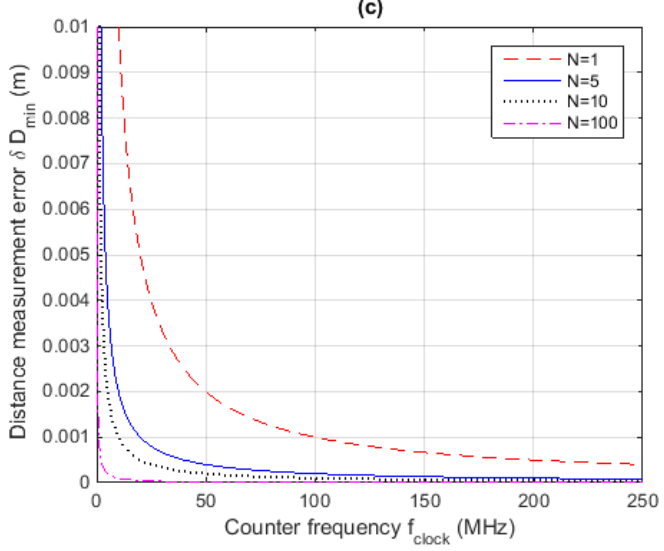

Fig. 3. Evolution, for $f_{e}=1 \mathrm{MHz}$, of (a) the distance measurement resolution $\delta D_{\min }$ against the heterodyning factor $r$, (c) against the counter frequency $f_{\text {clock }}$ for various averaging factors $N$ and of (b) the refresh rate $T_{r}$ against the heterodyning factor $r$ for various averaging factors $N$.

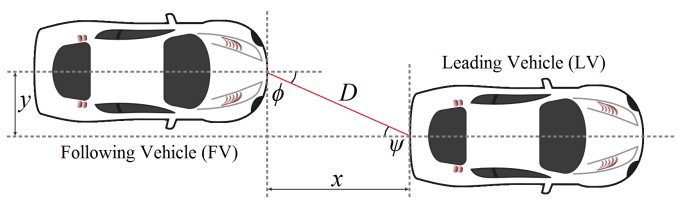

Fig. 4. Platooning configuration for FV to LV distance measurement simulations, where $\phi$ and $\psi$ represent respectively the irradiance and incidence angles and $D$ is the inter-vehicle distance. FV headlamps are represented as a single source only for ease of representation. where $\gamma$ is the PD responsivity, $h(t)$ is the channel impulse response and $n(t)$ is the AWGN. The channel frequency response $H(f)$ is considered flat in the frequency range of interest so that only the channel DC gain $H(0)$ matters. Vehicles headlamps and taillights have complex transmission beam patterns in order to meet international standards [19]. However, in this work, these light sources are considered Lambertian. In this case, the channel DC gain is defined as:

$$
H(0)=\frac{(m+1) A_{r}}{2 \pi D^{2}} \cos ^{m} \phi \cos \psi, \quad 0<\psi<\psi_{c},
$$

where $D, \phi$ and $\psi$ are respectively the inter-vehicle distance, the irradiance angle and the incidence angle, $A_{r}$ is the radiant sensitive area of the PD, $\psi_{c}$ is the PD field of view and $m$ is the order of Lambertian emission, defined as $m=-\ln 2 / \ln \left(\cos \phi_{1 / 2}\right)$, where $\phi_{1 / 2}$ is the semi-angle at half illuminance of the LED emitter. If the transmitted optical power is $P_{t}$, then the received light power $P_{r}$ will be:

$$
P_{r}=H(0) P_{t} .
$$

The signal power $\mathrm{S}$ at the receiver side will then be:

$$
S=\gamma^{2} P_{r}^{2}
$$

Different noise sources are introduced by the joint use of a PD and a transimpediance amplifier (TIA) for preamplification: shot noise, thermal noise, quantum noise, dark current noise or flicker noise. However, the two dominant noise sources are shot noise and thermal noise. Shot noise is induced by the incident luminous flux produced by the light source of interest and background light sources such as daylight, infrastructures light sources or vehicle lamps. Background light sources are usually dominant so shot noise is considered signal independent and thus modeled as a white Gaussian noise with variance $\sigma_{\text {shot }}^{2}$ :

$$
\sigma_{\text {shot }}^{2}=2 q \gamma P_{r} B+2 q \gamma I_{b g} I_{2} B,
$$

where $q$ is the electronic charge, $B$ is the equivalent noise bandwidth, $I_{b g}$ is the background photocurrent and $I_{2}$ is a noise bandwidth factor. The first term is thus the contribution of the light source of interest whereas the second term is the background noise. If daylight is the only background light considered, then $I_{b g}$ can be estimated to $5100 \mu \mathrm{A}$ in case of direct exposition or $740 \mu \mathrm{A}$ in case of indirect exposition [20]. Thermal noise is also signal independent since it is induced by charge carriers thermal agitation. Consequently, it is modeled as a white Gaussian noise with variance $\sigma_{\text {thermal }}^{2}$ :

$$
\sigma_{\text {thermal }}^{2}=\frac{8 \pi k T_{K}}{G} \eta A_{r} I_{2} B^{2}+\frac{16 \pi^{2} k T_{K} \Gamma}{g_{m}} \eta^{2} A_{r}^{2} I_{3} B^{3},
$$

where $k$ is Boltzmann's constant, $T_{K}$ is absolute temperature, $G$ is the open-loop voltage gain, $\eta$ is the fixed capacitance of photo detector per unit area, $\Gamma$ is the FET channel noise factor, $g_{m}$ is the FET transconductance and $I_{3}$ is a noisebandwidth factor. The total noise variance $N$ will then simply be the sum of shot noise and thermal noise:

$$
N=\sigma_{\text {shot }}^{2}+\sigma_{\text {thermal }}^{2} \text {. }
$$


Consequently, the signal-to-noise ratio (SNR) will be:

$$
S N R=\frac{S}{N}=\frac{\gamma^{2} H(0)^{2} P_{t}^{2}}{\sigma_{\text {shot }}^{2}+\sigma_{\text {thermal }}^{2}} .
$$

\section{System Performances}

TABLE I

Simulation Model Parameters

\begin{tabular}{|c|c|c|c|}
\hline Parameter & Value & Parameter & Value \\
\hline \multicolumn{2}{|c|}{ General parameters } & \multicolumn{2}{|c|}{ Channel parameters } \\
\hline$f_{e}$ & $1 \mathrm{MHz}$ & $\phi$ & $0^{\circ}$ \\
\hline$f_{\text {clock }}$ & $100 \mathrm{MHz}$ & $\psi$ & $0^{\circ}$ \\
\hline$r$ & 1500 & $I_{b g}$ & $740 \mu \mathrm{A}$ \\
\hline$N$ & 5 & $I_{2}$ & 0.562 \\
\hline \multicolumn{2}{|c|}{ Emitter parameters } & $B$ & $5 \mathrm{MHz}$ \\
\hline$P_{t}$ & $2 \times 1 \mathrm{~W}$ & $T_{K}$ & $298 \mathrm{~K}$ \\
\hline$\phi_{1 / 2}$ & $20^{\circ}$ & $\eta$ & $112 \mathrm{pF} / \mathrm{cm}^{2}$ \\
\hline \multicolumn{2}{|c|}{ Receiver parameters } & $\bar{G}$ & 10 \\
\hline$\gamma$ & $0.5 \mathrm{~A} / \mathrm{W}$ & $\Gamma$ & 1.5 \\
\hline$\overline{A_{r}}$ & $50 \mathrm{~mm}^{2}$ & $g_{m}$ & $30 \mathrm{mS}$ \\
\hline$\psi_{c}$ & $55^{\circ}$ & $I_{3}$ & 0.0868 \\
\hline
\end{tabular}

The different parameters and values used for simulations are listed in table I. These parameters correspond to a simple straight line configuration: both vehicle share the same longitudinal axis so the time delay from the right and left lamps to the receiver in both ways are equal. Each one of the four light sources considered has a power of 1 Watt. This value is obtained considering on the one hand that around $25 \%$ of the electrical input power is converted into light by a typical high-brightness LED [21] and on the other hand that a typical headlamp or taillight has an input power of around 15 Watt. In addition, the contribution from the light beams ground reflections are neglected. The frequency of emission is $1 \mathrm{MHz}$, which corresponds to a non-ambiguity range of around 150 meters. This distance is larger than the expected coverage of the system which means the nonambiguity range is not an issue in this case. Note that a larger frequency of emission would obviously be better in terms of measurement resolution but the white LED used in headlamps have a limited bandwidth of few megahertz that could only be enhanced through equalization techniques [22]. Note finally that the values of $r$ and $N$ are such that the theoretical measurement resolution is under 10 centimeters and the refresh rate is $267 \mathrm{~Hz}$.

First, an SNR analysis was conducted. Fig. 5(a) presents the SNR distribution in the vehicles emitters horizontal plane. The SNR is maximum in two points corresponding to the two light sources locations and is then degraded with lateral $X$ and longitudinal $Z$ distances. Fig. 5(b) represents the SNR evolution along the longitudinal direction $Z$ in the vehicle axis $(X=0)$. It clearly shows that the SNR remains higher than $15 \mathrm{~dB}$ up to 25 meters, which suggests that the signal received by the LV $s_{p^{\prime}}$ may be clearly detected without any other hard filtering than the bandwidth induced low-pass filtering. Consequently, the reconstructed square signal $s_{e^{\prime}}$ could be obtained by only amplifying and triggering $s_{p^{\prime}}$.

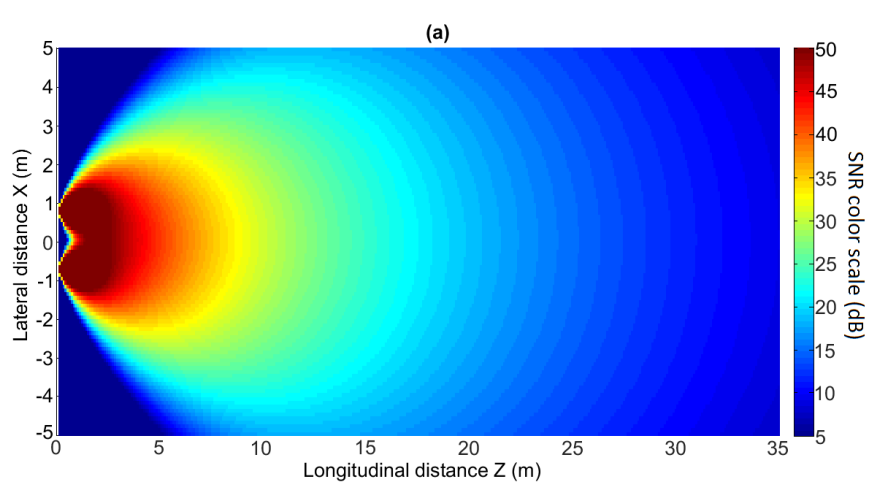

(b)

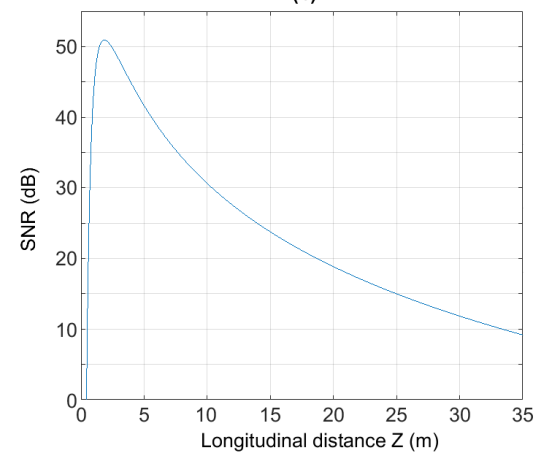

Fig. 5. (a) Horizontal distribution of the SNR of the received signal emitted by a vehicle according to the longitudinal $Z$ and lateral $X$ distance. (b) Detail of (a) in the vehicle axis $(X=0)$.

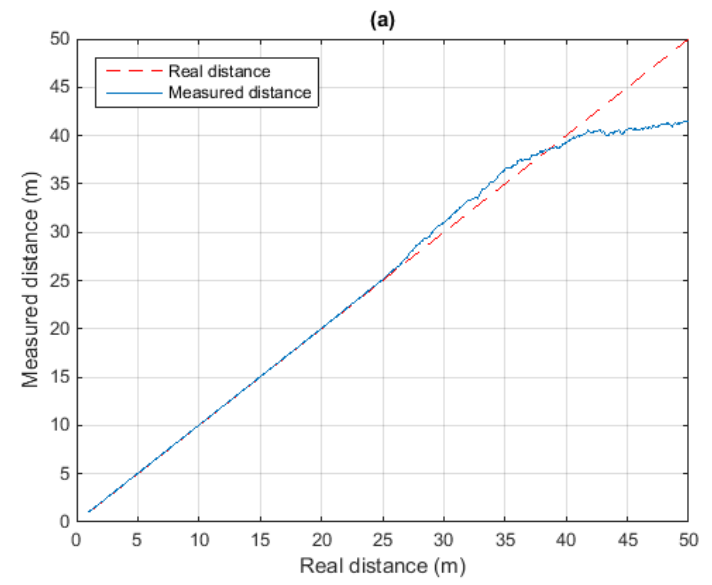

(b)

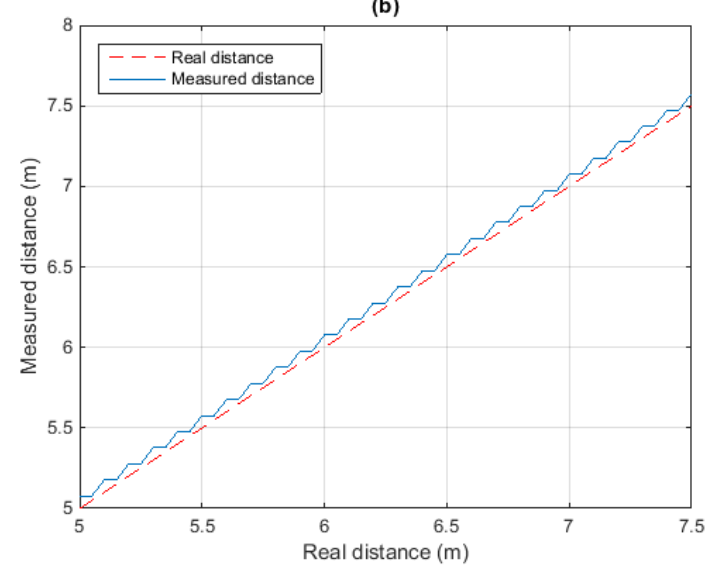

Fig. 6. (a) Evolution of the measured distance against the real distance and (b) detail of (a) between 5 and 7.5 meters, with 5 centimeters steps. 
Fig. 6(a) shows the evolution of the measured distance as the real distance increases when this method is used. From 1 meter to 25 meters, the estimated distance seems pretty close to the real one but after 25 meters, the measured distance diverges because the SNR is too low and the edges are not clearly identified anymore by a simple trigger. Fig. 6(b) represents the portion of Fig. 6(a) between 5 and 7.5 meters and shows that the distance measurement error is under 10 centimeters. This value is valid from 1 to 25 meters.

The measurement range can be extended by using more effective filtering. Fig. 7(a) shows the evolution of the measured distance against the real distance when second order Butterworth band-pass filtering with a passband of $400 \mathrm{kHz}$ centered on $1 \mathrm{MHz}$ is used. The resulting curve is increasing linearly from 1 to 50 meters but with a nonnegligible offset compared to the real distance. This offset is due to the additional phase-shifts introduced by the filtering on both vehicles and its evolution in represented on Fig. 7(b). It clearly remains rather constant up to 30 meters, which means it can be easily corrected by removing its mean value over this range. After 30 meters however, the offset starts to vary widely which would make this mean correction less reliable. This progressive performance degradation is due to the decreasing SNR that slowly prevents the filtering and triggering to work properly.

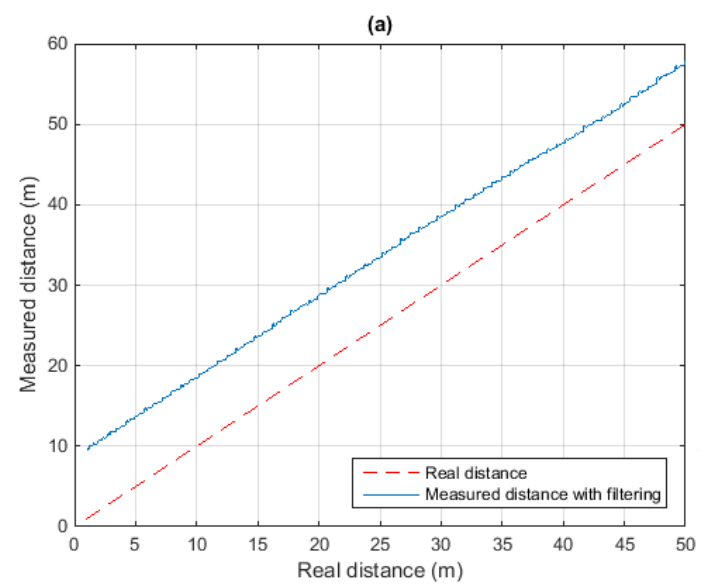

(b)

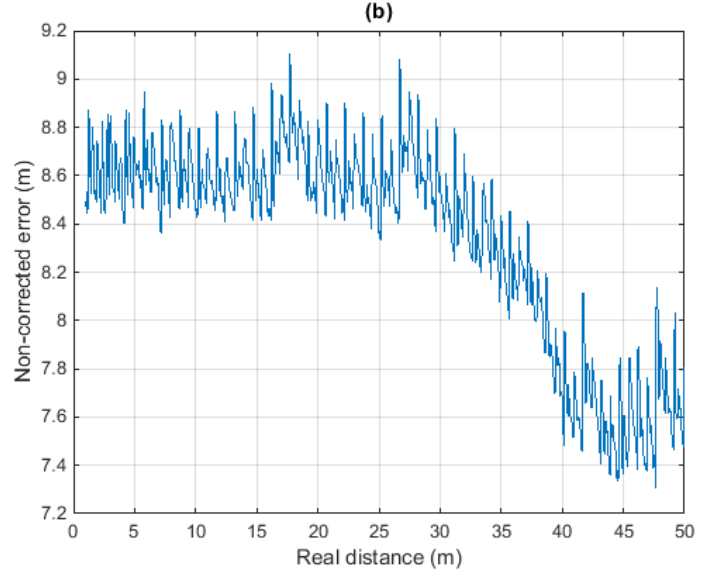

Fig. 7. Evolution of (a) the distance measured with filtering against the real distance with 5 centimeters steps and (b) the difference between the measured distance and the real distance.
Fig. 8(a) shows the distribution of the distance measurement error between 1 and 30 meters after the mean correction previously mentioned was applied. This histogram shows the error distribution is Gaussian-like. The corresponding Gaussian fit, given by the red curve, has zero mean value $\mu$ and standard deviation $\sigma=0.140$ meter. Consequently, the system resolution, defined as the $\pm \sigma$ confidence interval, is around 30 centimeters over 30 meters. Note however that given the relative linearity of the measures up to 50 meters, the mean correction could be used even though it would only provide a rough distance estimate with errors around 1 meter.

Finally note that a periodicity in the error can be highlighted through a Fast Fourier Transform (FFT) analysis. Fig. 8(b), which represents the magnitude of the FFT applied on the corrected measures between 1 and 30 meters, clearly shows a periodicity of 1.31 cycle/meter. In other words, the error reaches a peak every 76 centimeters. This periodicity is induced by the heterodyning method error, already detailed in Section II.D, which is emphasized by the filtering additional phase-shift.

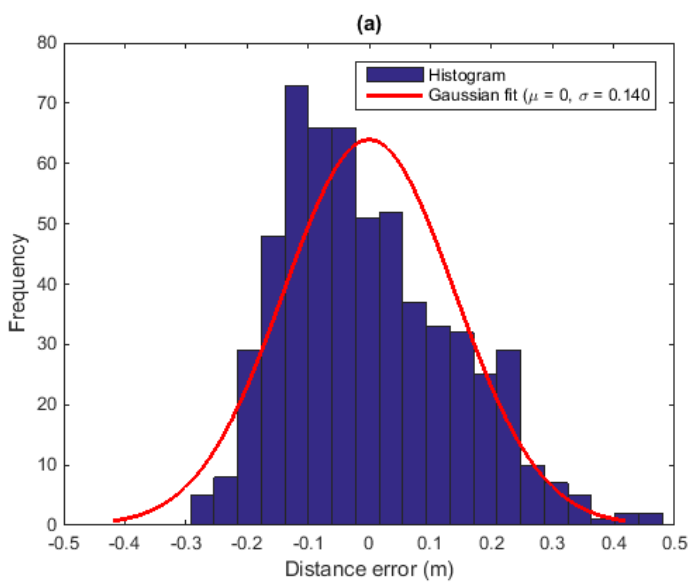

(b)

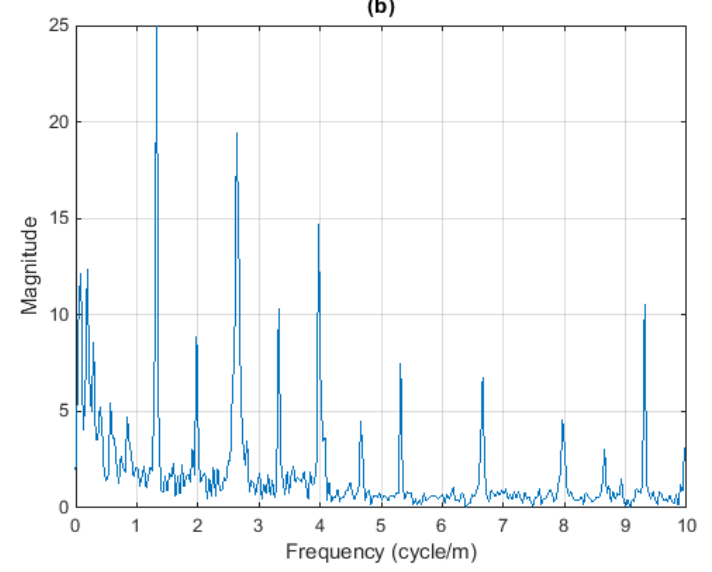

Fig. 8. (a) Histogram of the difference between the measured distance and real distance up to 30 meters corrected by removing the mean value of the offset over this range (blocs) and the corresponding Gaussian fit (red curve) and (b) FFT of this error. 


\section{CONCLUSION AND Future WORKS}

In this paper, we have presented a new concept of phase-shift rangefinder. Unlike classical infrared phase-shift rangefinder, our system uses the non-coherent visible light produced by headlamps and taillights to estimate the intervehicles distance.

A Simulink model of this system in a classical pathloss AWGN environment was implemented. Simulations show that our system is able to measure the absolute intervehicles distances up to 25 meters with a resolution under 10 centimeters, and up to 30 meters with 30 centimeters resolution at a refresh rate of $267 \mathrm{~Hz}$. The measurement range can be increased up to 50 meters but with an error over 1 meter.

Note that, unlike other techniques, the proposed method cannot provide the longitudinal and lateral inter-vehicles distance separately. However, the directivity of both headlamps and taillights on the one hand and the restricted FOV of the photodiode receivers on the other hand ensure a limited sensibility to interferences. In addition, the processing involved in each steps remains simple enough to make this system potentially low-cost. Consequently, it could be an interesting complement to existing solutions.

Future work will consist in the practical implementation and testing of this system in order to confirm the potential revealed by these simulations and prove it could be a reliable redundant sensor for platooning applications.

\section{REFERENCES}

[1] J. Wenger, "Automotive radar - status and perspectives," in Proc. IEEE CSIC'05, 2005, pp. 21-24.

[2] J. Lee, Y.-A. Li, M.-H. Hung, and S.-J. Huang, "A Fully-Integrated 77GHz FMCW Radar Transceiver in 65-nm CMOS Technology," IEEE J. Solid-State Circuits, vol. 45, no. 12, pp. 2746-2756, Dec. 2010.

[3] M. Goppelt, H.-L. Blcher, and W. Menzel, "Automotive radar investigation of mutual interference mechanisms," Adv. Radio Sci., vol. 8, pp. 55-60, Sep. 2010.

[4] C. Niclass, M. Soga, H. Matsubara, M. Ogawa, and M. Kagami, "A 0.18-um CMOS SoC for a 100-m-Range 10-Frame/s 200 96-Pixel Time-of-Flight Depth Sensor," IEEE J. Solid-State Circuits, vol. 49, no. 1, pp. 315-330, Jan. 2014.

[5] J. Schmackers, J. Velten, and A. Glasmachers, "Laser phase shift distance meter for vision based driver assistance systems," in Proc. IEEE IST, 2010, pp. 220-224.

[6] G. Kim, J. Eom, and Y. Park, "An Experiment of Mutual Interference between Automotive LIDAR Scanners," in Proc. 12th ITNG, 2015, pp. 680-685.

[7] J.-H. Wu, C.-C. Pen, and J.-A. Jiang, "Applications of the Integrated High-Performance CMOS Image Sensor to Range Finders from Optical Triangulation to the Automotive Field," Sensors, vol. 8, no. 3, pp. 1719-1739, Mar. 2008.

[8] R. Roberts, P. Gopalakrishnan, and S. Rathi, "Visible light positioning: Automotive use case," in Proc. IEEE VNC, 2010, pp. 309-314.

[9] S.-H. Yu, O. Shih, H.-M. Tsai, N. Wisitpongphan, and R. Roberts, "Smart automotive lighting for vehicle safety," IEEE Commun. Mag., vol. 51, no. 12, pp. 50-59, Dec. 2013.

[10] S. Tsugawa, "An overview on control algorithms for automated highway systems," in Proc. IEEE/IEEJ/JSAI ITSC, 1999, pp. 234-239.

[11] C. Bergenhem, S. Shladover, E. Coelingh, C. Englund, and S. Tsugawa, "Overview of platooning systems," in Proc. 19th ITS World Congress, 2012.

[12] P. S. Jootel. (2013, Jan.). SAfe Road TRains for the Environment - Final Report. SARTRE Project. U.K. [Online] Available: http://www.sartre-project.eu/en/publications/ Sidor/default.aspx.
[13] M. Y. Abualhoul, M. Marouf, O. Shagdar, and F. Nashashibi, "Platooning control using visible light communications: A feasibility study," in Proc. 16th IEEE ITSC, 2013, pp. 1535-1540.

[14] C.-Y. Wen, R. D. Morris, and W. A. Sethares, "Distance Estimation Using Bidirectional Communications Without Synchronous Clocking," IEEE Trans. Signal Process., vol. 55, no. 5, pp. 1927-1939, May 2007.

[15] B. Journet, G. Bazin, and F. Bras, "Conception of an adaptative laser range finder based on phase shift measurement," in Proc. 22nd IEEE IECON, 1996, vol. 2, pp. 784-789.

[16] S. Liu, J. Tan, and B. Hou, "Multicycle synchronous digital phase measurement used to further improve phase-shift laser range finding," Meas. Sci. Technol., vol. 18, no. 6, pp. 1756-1762, Jun. 2007.

[17] P. Hu, J. Tan, and H. Yang, "Phase-shift laser range finder based on high speed and high precision phase-measuring techniques," in Proc. 10th ISMTII, 2011.

[18] J. M. Kahn and J. R. Barry, "Wireless infrared communications," Proc. IEEE, vol. 85, no. 2, pp. 265-298, Feb. 1997.

[19] "Headlamps emitting an asymmetrical passing-beam.", UNECE ECE Regulation No. 112 - Rev.3, Jan. 2013.

[20] A. J. C. Moreira, R. T. Valadas, and A. M. de O. Duarte, "Optical interference produced by artificial light," Wirel. Netw., vol. 3, no. 2, pp. 131-140, May 1997.

[21] C. J. M. Lasance and A. Poppe, Thermal Management for LED Applications, New York, Springer, 2014.

[22] H. L. Minh, D. O'Brien, G. Faulkner, L. Zeng, K. Lee, D. Jung, and Y. Oh, " $80 \mathrm{Mbit} / \mathrm{s}$ Visible Light Communications using pre-equalized white LED," in Proc. 34th ECOC, 2008, pp. 1-2. 